\title{
Action spectroscopy for single-molecule motion induced by vibrational excitation with a scanning tunneling microscope
}

\author{
H. Ueba ${ }^{1}$ and B. N. J. Persson ${ }^{1,2}$ \\ ${ }^{1}$ Graduate School of Science and Engineering, Department of Electronics, University of Toyama, Gofuku, Toyama, Japan \\ ${ }^{2}$ IFF, FZ-Jülich, 52425 Jülich, Germany
}

(Received 8 November 2006; published 5 January 2007)

\begin{abstract}
We propose an action spectroscopy for single-molecule motion induced by vibrational excitation with a scanning tunneling microscope (STM). Calculations of the inelastic tunneling current for excitation of the C-O stretch mode of the $\mathrm{CO}$ molecule on metal surfaces are combined with a theory which describes how the energy in the vibrational mode is transferred to a reaction coordinate mode to overcome the activation barrier. The calculated rate for CO hopping on $\mathrm{Pd}(110)$ as a function of the bias voltage agrees with the experimental result. It is proposed that the second derivative of the reaction rate with respect to the bias voltage is related to the vibrational density of states, which usually cannot be directly observed in STM inelastic electron tunneling spectroscopy when a molecule motion is induced by vibrational excitation.
\end{abstract}

DOI: 10.1103/PhysRevB.75.041403

PACS number(s): 68.37.Ef, 68.43.Pq

Adsorbate motions on surfaces constitute the most fundamental step for many surface chemical reactions, because it is often the rate-limiting step for adsorbates to meet a reaction partner or to reach an active site before reaction takes place. The observation of adsorbates moving and making and breaking the bonds on surfaces provides indispensable information about the microscopic mechanisms of surface chemical reactions.

Tunneling electrons from a scanning tunneling microscope (STM) tip can be used as an atomic-size source of electrons for electronic and vibrational excitations and to manipulate individual atoms and molecules in a controlled manner across a surface. ${ }^{1-4}$ In 1998 Stipe et al. demonstrated that rotation of a single isolated acetylene molecule on $\mathrm{Cu}(100)$ is induced by vibrational energy transfer from the $\mathrm{C}-\mathrm{H}$ stretch mode excited by tunneling electrons to the frustrated rotational mode of the molecule. ${ }^{5}$ The rotation rate $R(V)$ exhibits a threshold at the bias voltage corresponding to the excitation of the $\mathrm{C}-\mathrm{H}$ stretch mode. The slope $d \log (R) / d V$ and the second derivative of the STM tunneling current in STM inelastic electron tunneling spectroscopy (IETS), $d^{2} I / d V^{2}$, both exhibit a peak at the same bias voltage $V$. This unambiguously demonstrates that the excitation of the $\mathrm{C}-\mathrm{H}$ stretch mode is a trigger for rotation. Singlemolecule manipulations have also been reported for hopping of $\mathrm{CO}$ on $\mathrm{Pd}(110),{ }^{6}$ and hopping and desorption of the $\mathrm{NH}_{3}$ molecule on $\mathrm{Cu}(100){ }^{7}$ where the excitation of the C-O stretch and $\mathrm{N}-\mathrm{H}$ stretch modes by tunneling electrons, respectively, have been found to be a trigger for their motions.

Recently Sainoo et $a l .{ }^{8}$ studied the vibrationally mediated motion of a single cis-2-butene molecule among four equivalent orientations on $\operatorname{Pd}(110)$. The close inspection of the reaction events per electron as a function of the applied bias voltage has enabled them to identify the vibrational modes involved in the orientational motion. A similar experiment has also been made for a $\mathrm{CH}_{3} \mathrm{~S}$ molecule hopping on $\mathrm{Cu}(111) .{ }^{9}$ The threshold of the reaction rate and the peak in its slope clearly indicate that excitation of the C-S stretch mode induces the hopping motion. Interestingly, they noticed that motion of cis-2-butene on $\operatorname{Pd}(110)$ is induced by the excitation of any of the vibrational modes $\nu(M-\mathrm{C}), \delta\left(\mathrm{CH}_{3}\right)$, $\nu(\mathrm{C}-\mathrm{C})$, and $\nu\left(\mathrm{CH}_{3}\right)$, but only $\nu(M-\mathrm{C})$ and $\nu\left(\mathrm{CH}_{3}\right)$ are detected in STM IETS.

Since the first STM IETS experiment ${ }^{10}$ for an acetylene molecule on $\mathrm{Cu}(100)$ there have been several issues. (1) Why are not all the vibrational modes that can be observed in infrared refection and absorption spectroscopy or electron energy loss spectroscopy observed in STM IETS? (2) Is there any selection rule? (3) What determines the line shape and intensity of the $d^{2} I / d V^{2}$ spectra? etc. Theories ${ }^{11-15}$ of STM IETS have made remarkable progresses since the first pioneering theory of STM IETS. ${ }^{16}$ Using the Keldysh Green's function method for an adsorbate-induced resonance coupled to the molecular vibration, we have shown that the vibration-induced modification to the tunneling current has both an elastic and an inelastic component. It is found that the elastic modification of the current yields always a reduction of the current, while the inelastic contribution gives an increase of the current. The competition between the negative elastic and positive inelastic components of the tunneling current leads to a decrease or increase in the conductance, and results in the dip or peak in the $d^{2} I / d V^{2}$ spectrum. ${ }^{13,14,17}$ Lorente and Persson ${ }^{11}$ also demonstrated that for $\mathrm{C}_{2} \mathrm{H}_{2}$ on $\mathrm{Cu}(100)$, due to the large negative elastic conductance of the $\mathrm{C}-\mathrm{C}$ mode, the tunneling conductance of the $\mathrm{C}-\mathrm{C}$ stretch mode is very small compared to that of the $\mathrm{C}-\mathrm{H}$ stretch mode. For all the modes except the $\mathrm{C}-\mathrm{H}$ stretch modes, the elastic and inelastic contributions tend to cancel and cannot be observed because of their weak signals. It is important to note that the $d^{2} I / d V^{2}$ signal in STM IETS is not a direct measure of the inelastic component of the tunneling current $I$, because the elastic component is also influenced by the electron-vibration interaction. ${ }^{13,16,17}$ The $d^{2} I / d V^{2}$ spectrum is not directly related to the vibrational density of states except under some special conditions, ${ }^{14}$ but it is determined by the adsorbate density of states coupled with the vibrational excitation by tunneling electrons through the adsorbate resonance. When the IETS $d^{2} I / d V^{2}$ spectrum shows a peak, ${ }^{18}$ its width observed in STM IETS is determined by modulation and thermal broadening in addition to the intrin- 
sic vibrational broadening. ${ }^{19,20}$ This makes it difficult to esimate the the vibrational broadening from the observed $d^{2} I / d V^{2}$ spectrum.

When a molecule motion is induced by a particular vibrational mode, it is difficult to observe it with STM IETS. This is indeed the case for $\mathrm{CO}$ hopping on metal surfaces. Lee and $\mathrm{Ho}^{21}$ clearly stated that on $\mathrm{Ag}(110) \mathrm{CO}$ moves when it is scanned at $0.25 \mathrm{eV}$, making it difficult to probe the $\mathrm{C}-\mathrm{O}$ stretch by STM IETS. On the other hand, vibrationally mediated motions are induced solely by the inelastic tunneling currents.

In this work we demonstrate that the action spectrum given by the second derivative of the reaction rate $R(V)$ with respect to a bias voltage provides direct access to the vibrational density of states of adsorbed molecules. We calculate the reaction rate $R(V)$ for $\mathrm{CO}$ hopping on a $\mathrm{Pd}(110)$ surface $^{6}$ using the theories of a single-electron process, ${ }^{22}$ inelastic tunneling currents, ${ }^{13}$ and the transition probability of deexciting the mode excited by tunneling electrons to the ground state while exciting the frustrated translation (FT) mode from its ground state to the higher-lying states above the barrier. ${ }^{6,23}$ A definition of the action spectroscopy has already been given in Ref. 5 as $d \log (R) / d V$ or in Ref. 8 as $R(V)$. In a analogy to the STM IETS spectrum given by $d^{2} I / d V^{2}$, we propose here that it is more appropriate to define $d^{2} R(V) / d V^{2}$ as the action spectrum of single-molecule motions.

We have developed a simple model to study the elementary single- and two-electron processes induced by vibrational mode coupling. ${ }^{22}$ Let $I_{i n}=\eta_{\nu} I$ ( $\eta_{\nu}$ is the inelastic tunneling fraction) be the inelastic tunneling current to excite the high-frequency (HF) $\nu$ mode with energy $\hbar \Omega$ to the first excited state, and $1 / \tau_{\nu}$ be the energy relaxation rate of the $\nu$ mode. The reaction rate of a single-electron process is given by $^{22}$

$$
R(V)=\frac{1}{\tau_{\nu, R C}} \tau_{\nu} I_{\text {in }}(V)
$$

where $1 / \tau_{\nu, R C}$ is the transition rate of deexciting the $\nu$ mode from its first excited state to the ground state while exciting the reaction coordinate (RC) mode (FT mode for hopping) with energy $\hbar \omega$ from its ground state to the higher-lying states above the reaction barrier $E_{B}$. We will denote $d^{2} R(V) / d V^{2}$ as the action spectrum. Our theory ${ }^{23}$ calculated the energy transfer from the $\nu$ mode to the FT mode via anharmonic coupling $\delta \omega$, in competition with the fast vibrational damping $1 / \tau_{\nu}$. This indirect process via the mode coupling illustrated in the inset in Fig. 1 gives the hopping rate

$$
R(V) \approx\left(\frac{\hbar \delta \omega}{E_{B}}\right)^{2} n^{3 / 2} e^{-2 n} I_{\mathrm{in}}(V),
$$

where $n=E_{B} / \hbar \omega$. The inelastic tunneling current $I_{\text {in }}$ is given by ${ }^{13,17}$

$$
I_{\mathrm{in}}=\frac{2 e}{\hbar} \frac{\Delta_{s} \Delta_{t}}{\Delta_{s t}^{2}} \int d \varepsilon \rho_{a}(\varepsilon) \Gamma_{a, p h}(\varepsilon)\left[n_{s}(\varepsilon)-n_{t}(\varepsilon)\right]
$$

where

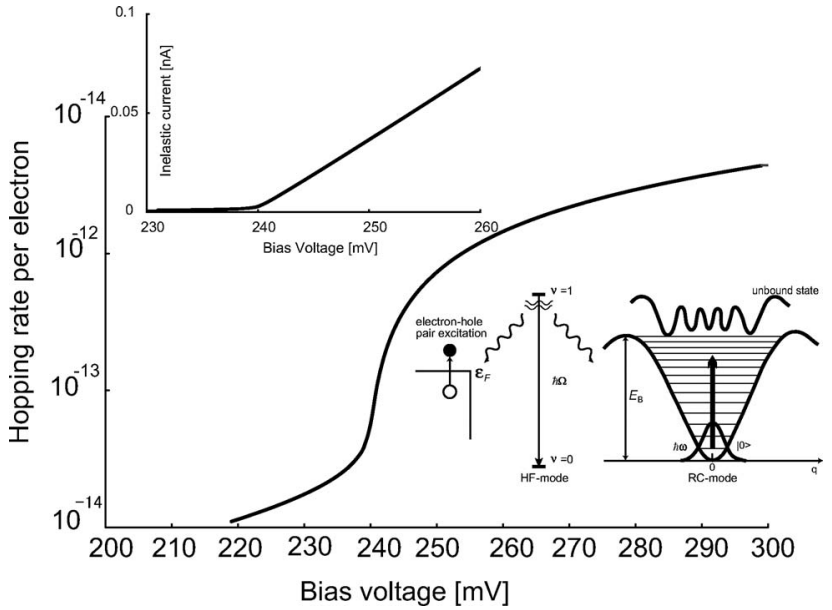

FIG. 1. Hopping rate $R(V)$ per electron for a $\mathrm{CO}$ molecule on $\operatorname{Pd}(110)$. See the text for parameters. Inset is the inelastic tunneling current $I_{i n}(V)$. The gradual increase below the threshold at $e V=240 \mathrm{mV}$ is due to a broadening of $\rho_{p h}(e V)$. Equation (2) is derived for the illustrated single-electron process, in which the decay of the $\nu$ mode from the excited state to the ground state excites the RC mode (FT mode for hopping) from the ground state to the higher-lying states above the barrier, in competition with the vibrational damping due to electron-hole pair excitation.

$$
\begin{aligned}
\Gamma_{a, p h}(\varepsilon)= & \pi \chi^{2} \int d \omega \rho_{p h}(\omega)\left\{n_{p h}(\omega) \times\left[\rho_{a}(\varepsilon-\omega)+\rho_{a}(\varepsilon+\omega)\right]\right. \\
& \left.+\left[1-n_{a}(\varepsilon-\omega)\right] \rho_{a}(\varepsilon-\omega)+n_{a}(\varepsilon+\omega) \rho_{a}(\varepsilon+\omega)\right\}
\end{aligned}
$$

describes the energy dissipation of the tunneling electrons to the vibrational excitation. Here $\Delta_{t(s)}$ is the adsorbatetip (substrate) coupling constant, $\Delta_{s t}=\Delta_{s}+\Delta_{t}, \quad n_{a}(\varepsilon)$ $=\left[\Delta_{s} n_{s}(\varepsilon)+\Delta_{t} n_{t}(\varepsilon)\right] / \Delta_{s t}$ is the effective distribution function for electrons, $n_{t(s)}(\varepsilon)=\left[\exp \left(\varepsilon-\mu_{t(s)}\right)+1\right]^{-1}$ with difference $\mu_{s}-\mu_{t}=e V$ fixed by the external bias voltage between the tip and the substrate, $n_{p h}(\omega)=1 /\left(e^{\hbar \omega / k_{B} T}-1\right)$ is the vibrational distribution function, $\rho_{a}(\varepsilon)=\Delta_{s t} / \pi\left[\left(\varepsilon-\varepsilon_{a}\right)^{2}+\Delta_{s t}^{2}\right]^{-1}$ and $\rho_{p h}(\omega)=\left(1 / \tau_{\nu} \pi\right)\left[(\omega-\Omega)^{2}+\left(1 / \tau_{\nu}\right)^{2}\right]^{-1}$ are the unperturbed adsorbate and vibrational densities of states, respectively. Since $\rho_{p h}(\omega)$ is peaked at $\omega=\Omega$ and $\rho_{a}\left(\mu_{s}-\omega\right)$ is a slowly varying function over the bias voltage for vibrational excitation, we can take $n_{p h}(\omega)=0$ and $n_{a}(\varepsilon)$ $\simeq \Delta_{t} / \Delta_{s t} \theta\left(\mu_{t}-\varepsilon\right)+\Delta_{s} / \Delta_{s t} \theta\left(\mu_{s}-\varepsilon\right)$ at low $T$. We then obtain

$$
\frac{d^{2} I_{\text {in }}}{d V^{2}} \simeq \frac{2 \pi e^{3}}{\hbar} \frac{\Delta_{s} \Delta_{t}^{2}}{\Delta_{s t}^{3}} \chi^{2} \rho_{a}\left(\mu_{s}\right) \rho_{a}\left(\mu_{s}-e V\right) \rho_{p h}(e V) .
$$

This reveals that the action spectrum defined by $d^{2} R(V) / d V^{2}$ is directly proportional to $\rho_{p h}(\mathrm{eV})$.

Figure 1 shows the $\mathrm{CO}$ hopping rate per electron calculated for the parameters $\hbar \Omega=240 \mathrm{meV}, \hbar \omega=25 \mathrm{meV}, E_{B}$ $=150 \mathrm{meV}, \hbar \delta \omega=5 \mathrm{meV}, n=6, \varepsilon_{a}=2.0 \mathrm{eV}, \chi=0.3 \mathrm{eV}$. This set of parameters corresponds to $\mathrm{CO}$ on $\mathrm{Pd}(110)$, and gives a vibrational lifetime of about 1 ps. Also plotted in Fig. 1 is $I_{\text {in }}$ calculated by Eq. (3) at low T. The gradual increase below the threshed at $e V=\hbar \Omega$ is due to a broadening of $\rho_{p h}(e V)$. 


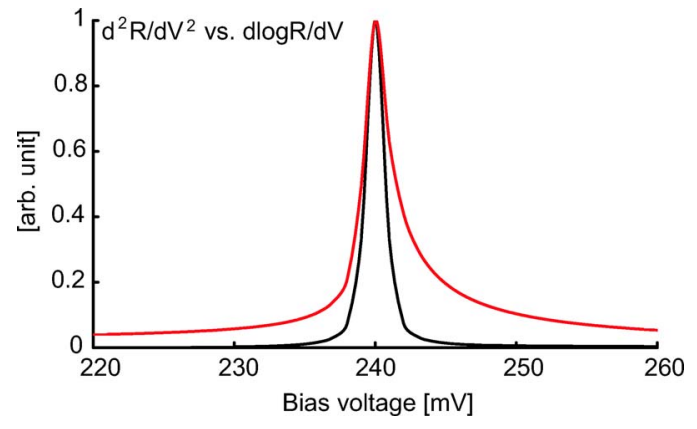

FIG. 2. (Color online) The action spectrum $d^{2} R(V) / d V^{2}$ (black curve), and $d \log R / d V$ (red/dark gray curve) calculated from $R(V)$ in Fig. 1. Each curve is normalized to its maximum value.

Even at this level of the simplified model calculation, we find a nice agreement with the experimental result. ${ }^{6}$ Figure 2 shows the action spectra $d^{2} R(V) / d V^{2}$ and $d \log (R) / d V$ for comparison. As explicitly derived in Eq. (5) the action spectrum exhibits a peak at $e V=\hbar \Omega$ with the width given by the vibrational broadening. This demonstrates that the action spectrum directly reflects the vibrational density of states, which cannot be accessible with STM IETS. Stipe et al. ${ }^{5}$ reported $R(V)$ and $d \log (R) / d V$ for rotation of $\mathrm{C}_{2} \mathrm{H}_{2}$ on $\mathrm{Cu}(100)$. They remarked that the derivatives of $R(v)$ show a narrow voltage range over which the increase in rotation rates occurs. The voltage corresponds to the peak position observed at $e V=\hbar \Omega=358 \mathrm{meV}$ (the excitation of the $\mathrm{C}-\mathrm{H}$ stretch mode) in STM IETS. For rotation of a single $\mathrm{C}_{2} \mathrm{H}_{2}$ molecule between two diagonal sites on the $\mathrm{Cu}(100)$ surface the molecule remains at the same adsorption site, so that the $\mathrm{C}-\mathrm{H}$ mode can be detected with IETS. However, this is not a case for hopping or desorption of a molecule because it moves away under the STM tip once it is scanned at the bias voltage exciting the vibrational mode which induces the motion, as in the case of CO hopping on Ag (110) (Ref. 21) and $\mathrm{Pd}(110)$ (Ref. 6) as well as $\mathrm{NH}_{3}$ hopping and desorption on $\mathrm{Cu}(100) .{ }^{7}$ The asymmetric tail observed above the threshold in $d \log (R) / d V$ [see Fig. 4(b) in Ref. 5 for rotation of $\mathrm{C}_{2} \mathrm{H}_{2}$ ] is reproduced in Fig. 2. A similar behavior was also abserved for the N-H stretch mode, which induces hopping of $\mathrm{NH}_{3}$ on $\mathrm{Cu}(100){ }^{7}$ Such an asymmetry does not appear in $d^{2} R(V) / d V^{2}$.

Our proposal can also be applied to a two-electron process such as desorption of $\mathrm{NH}_{3}$ from $\mathrm{Cu}(100),{ }^{7}$ where the reaction rate is given by $R(V)=\left(1 / \tau_{2 \nu, R C}\right) \tau_{\nu} I_{i n}^{2}(V)$ in terms of the excitation rate of the reaction-coordinate mode $1 / \tau_{2 \nu, R C}$ via the two times decay of the N-H stretch mode excited by tunneling electrons and the vibrational relaxation time $\tau_{\nu}{ }^{22,24}$ and to multielectron processes $R(V) \propto I^{n}(V) / \gamma^{n-1}$ for vibrational ladder climbing following a direct excitation of the $\mathrm{RC}$ mode [here, $n$ stands for the number of vibrational levels (=tunneling electron excitations) in the $\mathrm{RC}$ potential well]. ${ }^{25,26}$ Our proposal of action spectroscopy is valid for the adsorbate motions induced by direct and indirect excitation of the RC mode. For the direct process the action spectrum reflects the vibrational density of states $\rho_{p h}(\mathrm{eV})$ of the $\mathrm{RC}$ mode, while for the indirect one it gives $\rho_{p h}(\mathrm{eV})$ of the vibrational mode, such as the $\mathrm{C}-\mathrm{O}$ or $\mathrm{N}-\mathrm{H}$ stretch mode excited by tunneling electrons and anharmonically coupled to the RC mode. In both cases the IETS spectrum does not always give $\rho_{p h}(\mathrm{eV})$ because of the elastic correction in the IETS spectrum.

The single-Co-atom hopping rate $^{27}$ on $\mathrm{Cu}(111)$ has been theoretically studied by Liu and Gao. ${ }^{28}$ Simulation using a power law, compared with experiment, suggests that the atom hopping is due to the local heating of the FT mode. The frequency and lifetime of the FT mode can be extracted from the second derivative of the power law of the hopping rate. The line shape of the action spectrum is directly related to the vibrational density of states, and the width of this action spectrum gives the intrinsic vibrational broadening. Recently Liu and Gao reported a theoretical calculation of the vibrational spectrum and the lifetime of $\mathrm{Co}$ on the $\mathrm{Cu}(111)$ surface. ${ }^{29}$ The calculated lifetime $(1.6 \mathrm{ps})$ of the FT mode may be compared to the width of the action spectrum for Co hopping.

STM allows the real-space observation of site-resolved single-molecule motions. The reaction rate as a function of applied bias voltage and tunneling currents provides important information about the elementary processes of electronic or vibrational excitation by tunneling electrons. When excitation of a particular vibrational mode is a trigger for molecule hopping or desorption, it cannot be usually observed by STM IETS. Here, we have proposed that, since the motion is induced by inelastic tunneling electrons, the second derivative of the reaction rate with respect to the bias voltage provides a direct access of the vibrational density of states. The width of this action spectrum gives the intrinsic vibrational broadening of a single adsorbed molecule on a solid surface. The identification of the vibrational mode responsible for various surface motions and reactions is essential for achieving mode-selective control of chemical reactions. A combination of dynamic spectroscopic techniques such as action spectroscopy and "static" techniques such as STM IETS, gives important information to establish the dynamics related to the electron-vibration coupling in molecules on surfaces. ${ }^{8}$

We would like to thank Maki Kawai and Y. Kim for sharing the idea of action spectroscopy and for discussions. H.U. was supported by a Grant-in-Aid for Scientific Research of Priority Areas ("Electron Transport Through a Linked Molecule in Nanoscale") from the Ministry of Education, Science and Culture of Japan, and a Grant-in-Aid for Scientific Research (Grant No. B-18340085) from JSPS. B.N.J was financially supported by JSPS (program S-06022). 
${ }^{1}$ W. Ho, J. Chem. Phys. 117, 11033 (2002).

${ }^{2}$ S.-W. Hla and K.-H. Rieder, Annu. Rev. Phys. Chem. 54, 307 (2003)

${ }^{3}$ H. Ueba, Surf. Sci. 10, 771 (2003); H. Ueba and B. N. J. Persson, Surf. Sci. 566-568, 1 (2004).

${ }^{4}$ A. J. Mayne, G. Dujardin, G. Comtet, D. Riedel, Chem. Rev. (Washington, D.C.) (to be published).

${ }^{5}$ B. C. Stipe, M. A. Rezaei, and W. Ho, Phys. Rev. Lett. 81, 1263 (1998).

${ }^{6}$ T. Komeda, Y. Kim, M. Kawai, B. N. J. Persson, and H. Ueba, Science 295, 2055 (2002).

${ }^{7}$ J. I. Pascual, N. Lorente, Z. Song, H. Conrad, and H.-P. Rust, Nature (London) 423, 525 (2003).

${ }^{8}$ Y. Sainoo, Y. Kim, T. Okawa, T. Komeda, H. Shigekawa, and M. Kawai, Phys. Rev. Lett. 95, 246102 (2005).

${ }^{9}$ M. Ohara, Y. Kim, and M. Kawai, Jpn. J. Appl. Phys., Part 1 45, 2022 (2006).

${ }^{10}$ B. C. Stipe and W. Ho, Science 280, 1733 (1998).

${ }^{11}$ N. Lorente and M. Persson, Phys. Rev. Lett. 85, 2997 (2000).

${ }^{12}$ N. Mingo and K. Makoshi, Phys. Rev. Lett. 84, 3694 (2000).

${ }^{13}$ T. Mii, S. G. Tikhodeev, and H. Ueba, Surf. Sci. 602-503, 26 (2002).

${ }^{14}$ T. Mii, S. G. Tikhodeev, and H. Ueba, Phys. Rev. B 68, 205406
(2003).

${ }^{15}$ M. Galperin, M. A. Ratner, and A. Nitzan, J. Chem. Phys. 121, 11965 (2004).

${ }^{16}$ B. N. J. Persson and A. Baratoff, Phys. Rev. Lett. 59, 339 (1987).

${ }^{17}$ H. Ueba, T. Mii, and S. G. Tikhodeev (unpublished).

${ }^{18}$ The IETS $d^{2} I / d V^{2}$ spectra exhibit peak, dip or derivativelike line shapes depending on the positions of the adsorbate states. See Refs. 14 and 15 for more details.

${ }^{19}$ L. J. Lauhon and W. Ho, Rev. Sci. Instrum. 72, 216 (2001).

${ }^{20}$ W. Wang, T. Lee, I. Kretzschmar, and M. A. Reed, Nano Lett. 4, 643 (2004)

${ }^{21}$ H. J. Lee and W. Ho, Science 286, 1719 (1999).

${ }^{22}$ H. Ueba, T. Mii, N. Lorente, and B. N. J. Peersson, J. Chem. Phys. 123, 084707 (2005).

${ }^{23}$ B. N. J. Persson and H. Ueba, Surf. Sci. 502-503, 18 (2002).

${ }^{24}$ N. Lorente, J. I. Pascual, and H. Ueba, Surf. Sci. 593, 122 (2005).

${ }^{25}$ S. Gao, M. Persson, and B. I. Lundqvist, Phys. Rev. B 55, 4825 (1997).

${ }^{26}$ S. G. Tikhodeev and H. Ueba, Phys. Rev. B 70, 125414 (2004).

${ }^{27}$ J. A. Stroscio and R. J. Celotta, Science 306, 242 (2004).

${ }^{28}$ K. Liu and S. Gao, Phys. Rev. Lett. 95, 226102 (2005).

${ }^{29}$ K. Liu and S. Gao, Phys. Rev. B 74, 195433 (2006). 